\title{
To perceive a perfect Dora Maar in peripheral vision
}

\author{
Rong-Ding $\mathrm{He}^{1 \dagger}$, Tao $\mathrm{Su}^{2 \dagger}$, Yang $\mathrm{Yang}^{1}$, Ying $\mathrm{Liu}^{2}$, Yan $\mathrm{Wei}^{2}$ \& Rong-Qiao $\mathrm{He}^{2 *}$ \\ ${ }^{1}$ Fine Art College, Chengdu University, Chengdu 610106, China; \\ ${ }^{2}$ State Key Laboratory of Brain and Cognitive Science, Institute of Biophysics, Chinese Academy of Sciences, Beijing 100101, China;
}

Received November 30, 2015; accepted February 16, 2016; published online April 6, 2016

Citation: He, R.D., Su, T., Yang, Y., Liu, Y., Wei, Y., and He, R.Q. (2016). To perceive a perfect Dora Maar in peripheral vision. Sci China Life Sci 59, 640-642. doi: 10.1007/s11427-016-5031-8

\section{Dear Editor,}

The Portrait of Dora Maar $(92 \mathrm{~cm} \times 65 \mathrm{~cm})$ painted by Pablo Picasso (1937) is an aesthetic masterpiece in the world (Brigitte, 1996). Picasso paints Dora seating on a chair in frontal view with an off-set profile and disjunctive facial features (André, 1976): that is, Dora's nose becomes an extension of her face on which the "far" eye is seen looking straight at us. Why did Picasso paint his lover Dora in such a tortured style?

As we know, peripheral vision, as opposed to central vision, occurs outside the center of gaze (He and He, 2014). Identifying objects from the corner of one's eye is possible in isolation but difficult in a cluttered environment (Yeotikar et al., 2011). An inability to recognize objects resulted from clutter in peripheral vision is a phenomenon known as "crowding" (Bi et al., 2009). Here, we uncover how the inner-outer asymmetry (Whitney and Levi, 2011) of crowding effect on our perception of the disjunctive human appearance results in a perfect illusion in peripheral vision.

To understand the disjunction of Dora, we considered the portrait in different ways. Firstly, looking directly at Dora's face, one sees her tortured appearance in frontal view. But, when the sight line is shifted away from her face and to the fixation point as indicated in Figure S1, a miracle seems to occur. The disjointed face is transformed in the illusion of a normal and beautiful face in the peripheral visual field. Dora's cheek appears vivid and round, forming a 3D-like im-

$\dagger$ Contributed equally to this work

*Corresponding authors (email: herq@sun5.ibp.ac.cn) age in our visual background. Picasso painted Dora on a 2D canvas, and was apparently able to perceive her 3D-like appearance in peripheral vision.

Peripheral vision is the work of the rods, nerve cells located largely outside the macula (the center) of the retina. The rods are also responsible for night vision and low-light vision but they are insensitive to color (Curcio et al., 1990). We decolorized the portrait of Dora (black and white) to prevent the oil colors from interfering with observation (Figure S2 in Supporting Information). To demonstrate the effect of peripheral vision, we recruited 30 participants (15 male; 15 female). First, each participant looked at Dora on screen from the frontal view for $5 \mathrm{~s}$ (Figure S2a in Supporting Information). A group of three portraits was then shown to participants (Figure S3, i, ii, iii, iv, v and vi, randomly assigned in Supporting Information) and they were asked to select the portrait that best matched what they had seen in their foveal vision. Each of them picked the tortured Dora out (Figure 1A, T). This, as a control task, confirmed that all the participants had normal vision and visual processing to recognize Dora's tortured appearance.

Participants were then asked to move their foveal vision onto the fixation point as shown in Figure S2b, and stare at the point for $5 \mathrm{~s}$, to perceive Dora in the peripheral field. Participants were then asked to select from a random group of three portraits what they had seen in peripheral vision (Figure S3 in Supporting Information). Twenty-seven participants picked the "normal" Dora out (Figure 1A, N). Three participants hesitated in their selection (Figure 1A, $\mathrm{H})$. However, after they were explained how to perceive the portrait with their peripheral vision again, these three par- 

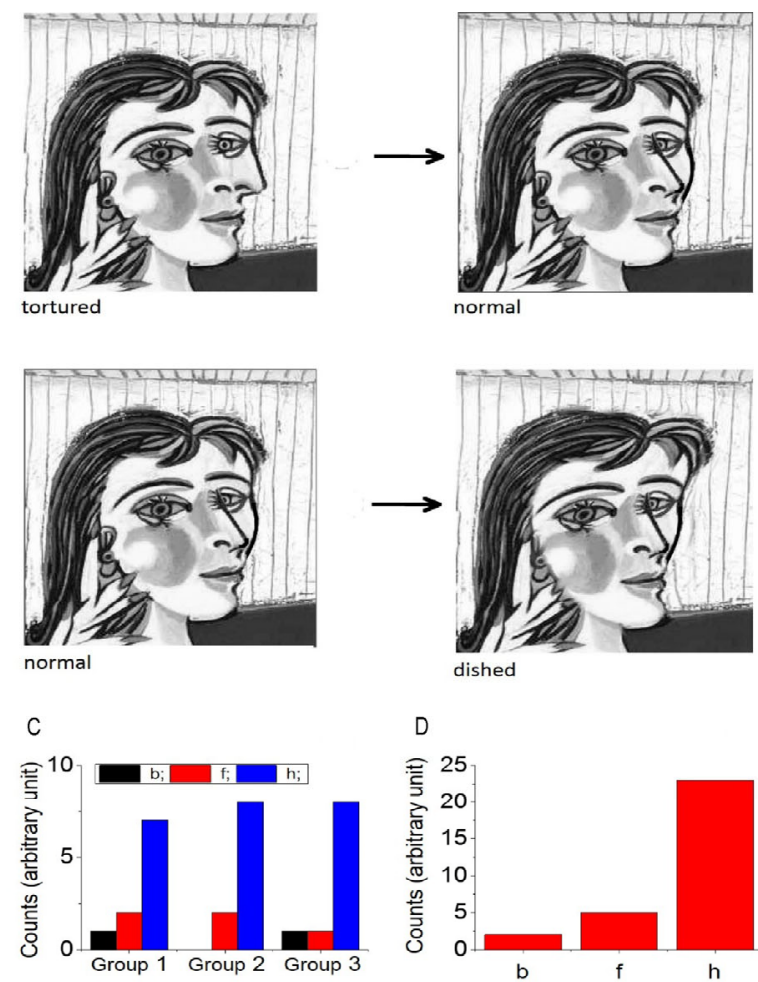

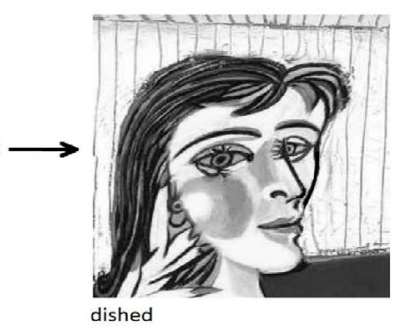

D

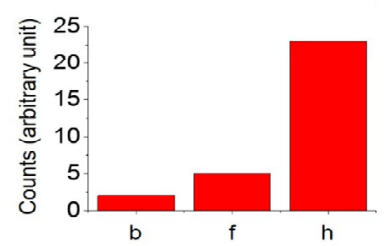

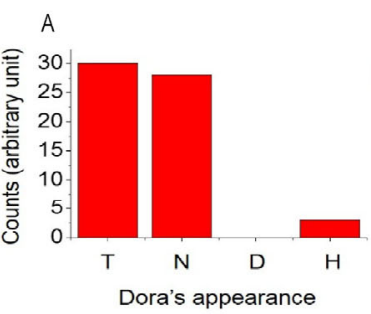

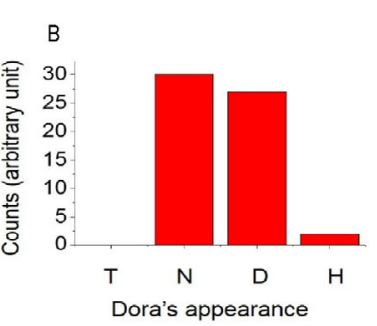

$\mathrm{E}$

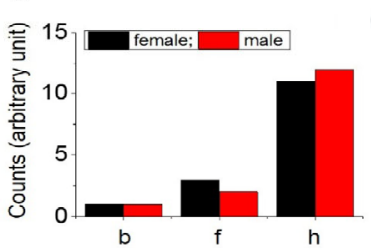

Figure 1 From tortured to normal appearance with peripheral vision. Different Dora portraits in tortured (T), normal (N) and dished (D) appearances were respectively used in the peripheral visual tasks as described in Supplementary Information. A, Participants were asked to look directly at Dora in Figure S2, and choose the matching image from one of the three portraits in Figure S3. All participants picked the tortured portrait out (T). Then, they were asked to move their foveal sight onto the fixation point to perceive Dora in their peripheral vision. 28 of them picked normal portrait out (N) and two of them hesitated $(\mathrm{H})$ to give their answer in the task. B, When the normal Dora was presented in frontal view, all participants correctly identified the image (N). 27 participants perceived a dished Dora (D) on peripheral view when the normal Dora was used in frontal view. Three of them hesitated to pick the portrait out (H). Participants $(n=30)$ were divided into three groups and asked to look a perfect circle at the fixation point for $5 \mathrm{~s}$ (Figure S5B). The three groups were requested to identify the objects from questionnaires in Figures S6, S7 or S8. They were asked to choose the object most similar to what they had perceived in peripheral vision. Objects identified by three groups (C), all participants (D), female and male participants (E) are depicted as indicated, respectively.

ticipants also chose the normal Dora. No participants chose the dished portrait (Figure 1A, D). Note that any attempting the subjects to choose the option is not allowed. These results point to the $3 \mathrm{D}$-like illusion that results from perception in peripheral vision of the disjointed Dora.

In order to investigate why peripheral vision results in the disjointed Dora being perceived as having a normal facial structure, we used a portrait of the normal Dora as an examinational object (Figure S4a in Supporting Information). As a control task, all participants confirmed that the Dora perceived in their foveal vision was the normal Dora, and not the tortured one (Figure 1B, N). However, when perceived in the peripheral visual field using the fixation point (Figure S4b in Supporting Information), 28 participants selected the dished portrait of Dora as the image that best matched the one they had perceived (Figure 1B, D), a portrait in which Dora's left cheek has a concave appearance. Two of them hesitated to select an image (Figure 1B, H). They, however, also chose the dished portrait after having the task re-explained. No participants selected the tortured portrait at this time (Figure 1B, T). The concave illusion of an object perceived in peripheral vision may be resulted from the inner-outer asymmetry of crowding ef- fect (Whitney and Levi, 2011). Picasso's precise rendering of Dora Maar in a tortured structure compensates the eccentricity (asymmetry) of human peripheral vision (Levi and Carney, 2009; Strasburger et al., 2015).

To clarify whether the asymmetry effect is related with the perception as described by Whitney and Levi (Whitney and Levi, 2011), participants were asked to look at a circle on the screen in the peripheral field (Figure S5a in Supporting Information). The object observed in foveal visual resulted in all participants correctly identifying a perfect circle. After looking at the circle, different shapes were shown to participants on a questionnaire and they were asked to choose which one was most similar to what they had perceived in peripheral vision (Figure S5b in Supporting Information). To exclude interference by the recognition of object positions, we divided the participants into three groups (10 people in each). Three separate questionnaires (Figures S6, S7 and S8 in Supporting Information) were used for the first, second and third group, respectively. When asked to select what they had seen in peripheral vision (Figure 1C), 23, five and two of the participants identified object $h$, object $f$ and object $b$ (Figure 1D), respective- 
ly. Object h was most selected by participants in all groups (horizontal axis was suppressed to $\sim 85 \%$ compared with the perfect circle). No participants picked the other objects (a, c, $\mathrm{d}, \mathrm{e}, \mathrm{g}$, and i) under the trial conditions. No significant difference was observed between male and female in their peripheral visual perceptions $(P>0.05)$ (Figure 1E). The three objects ( $h, f$, and $b$ ) were concaved at the nasal side of the circle but not the temporal side. This demonstrated that the eccentricity occurs in the nasal peripheral field, which is the same side as Dora's disjointed nose and left eye. Picasso painted the tortured Dora with a wider face at our nasal side, which counteracts the asymmetry perception with peripheral vision.

Picasso painted the Portrait of Dora Maar with thick lines, clear-cut structure, and in bright color, which lets us perceive a vivid Dora (Shen et al., 2015). He gives Dora an extended nose and cheek to compensate for the asymmetry crowding effect of peripheral vision as described by Whitney and Levi (Whitney and Levi, 2011). However, as we think, the mechanism should be more complicated. The poor spatial resolution in peripheral vision and surround suppression may be also involved in the perception of the disjunctive Dora. The detailed mechanism needs to be further investigated. With a master's talent, on a 2D Picasso has painted a portrait that appears vivid, illusively full and 3D-like appearance in peripheral vision. In short, whether peripheral vision results in the illusory perception of a normal object depends upon the structure of the visual object.
Compliance and ethics The author(s) declare that they have no conflict of interest.

Acknowledgements We thank all the participants from the Institute of Biophysics, Chinese Academy of Sciences and Chengdu University for their collaboration in this work. Ms. Donna Lu (Queensland Brain Institute, The University of Queensland, Brisbane, Australia) edited the language of this paper. This work was supported by the External Cooperation Program of BIC, Chinese Academy of Sciences (GJHZ201302): Collaboration between Institute of Biophysics CAS and Queensland Brain Institute, University of Queensland, Australia.

Brigitte, L. (1996). Portraits of Dora Maar. Picasso and Portraiture. (New York: Harry N Abrams Inc), pp. 395-396.

He, R.D., and He, R.Q. (2014). What did the bronze eye-like prism tell us? Sci Chin Life Sci 57, 445-447.

Yeotikar, N.S., Khuu, S.K., Asper, L.J., and Suttle, C.M. (2011). Configuration specificity of crowding in peripheral vision. Vision Res 51, 1239-1248.

Bi, T.Y., Cai, P., Zhou, T.G., and Fang, F. (2009). The effect of crowding on orientation-selective adaptation in human early visual cortex. $\mathrm{J}$ Vis $9,1-10$.

Whitney, D., and Levi, D.M. (2011). Visual crowding: a fundamental limit on conscious perception and object recognition. Trend Cogn Sci 15, $160-168$.

Curcio, C.A., Sloan, K.R., Kalina, R.E., and Hendrickson, A.E. (1990). Human photoreceptor topography. J Comp Neurol 292, 497-523.

Levi, D.M., and Carney, T. (2009). Crowding in peripheral vision: why bigger is better. Cur Biol 19, 1988-1993.

Strasburger, H., Rentschler, I., and Juttner, M. (2015). Peripheral vision and pattern recognition: a review. $\mathrm{J}$ Vis $11,13$.

Shen, J., Peli, E., and Bowers, A.R. (2015). Peripheral prism glasses: effects of moving and stationary backgrounds. Optom Vis Sci 92, 412-420.

André, M. (1976). Picasso's Mask (Eng., March 22, 1995). Reissue edition. (New York: Holt, Rinehart and Winston), pp. 138-143.

Open Access This article is distributed under the terms of the Creative Commons Attribution License which permits any use, distribution, and reproduction in any medium, provided the original author(s) and source are credited.

\section{SUPPORTING INFORMATION}

Figure S1 A perfect Dora imaging in the peripheral vision.

Figure S2 Tortured Dora in black and white.

Figure S3 Dora with different structural faces in different groups.

Figure S4 Normal Dora in black and white.

Figure S5 A circle designed for peripheral vision.

Figure S6 Objects for participants to recognize what they saw in their peripheral vision.

Figure S7 Objects for participants to recognize what saw in their peripheral vision.

Figure S8 Objects for participants to recognize what they saw in their peripheral vision.

The supporting information is available online at life.scichina.com and link.springer.com. The supporting materials are published as submitted, without typesetting or editing. The responsibility for scientific accuracy and content remains entirely with the authors. 